\title{
OTEC Thermal Resource Report for Dampier Land, Australia
}

May 1979

Prepared for

U.S. Department of Energy

Assistant Secretary for Energy Technology

Division of Central Solar Technology

Under Contract No. ET-78-C-01-2898 


\section{DISCLAIMER}

This report was prepared as an account of work sponsored by an agency of the United States Government. Neither the United States Government nor any agency Thereof, nor any of their employees, makes any warranty, express or implied, or assumes any legal liability or responsibility for the accuracy, completeness, or usefulness of any information, apparatus, product, or process disclosed, or represents that its use would not infringe privately owned rights. Reference herein to any specific commercial product, process, or service by trade name, trademark, manufacturer, or otherwise does not necessarily constitute or imply its endorsement, recommendation, or favoring by the United States Government or any agency thereof. The views and opinions of authors expressed herein do not necessarily state or reflect those of the United States Government or any agency thereof. 


\section{DISCLAIMER}

Portions of this document may be illegible in electronic image products. Images are produced from the best available original document. 


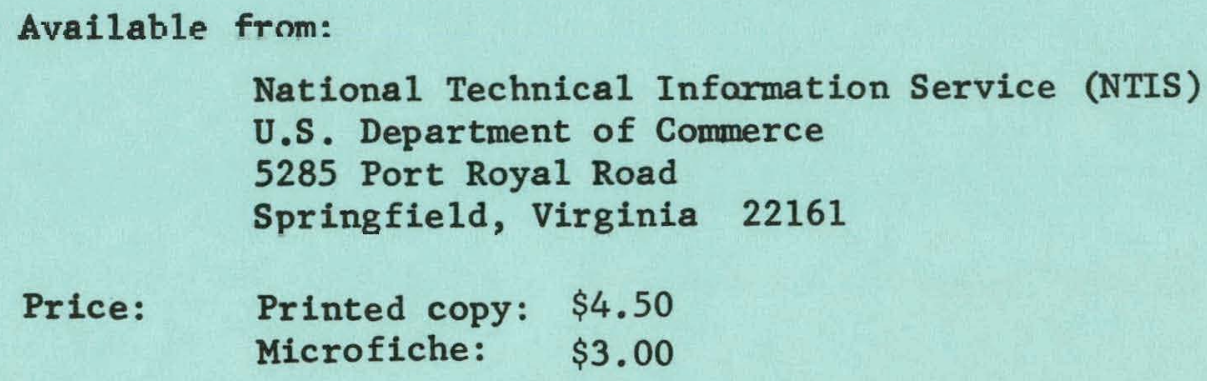




\section{OTEC Thermal Resource Report for Dampier Land, Australia}

May 1979

Prepared for

U.S. Department of Energy

Assistant Secretary for Energy Technology

Division of Central Solar Technology

Washington, D.C. 20585

Prepared by

William Alan Wolff

Ocean Data Systerns, Inc.

Monterey, California 93940

Under Contract No. ET-78-C-01-2898

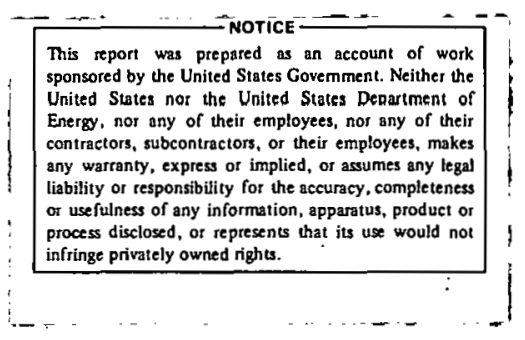




\section{NOTICE}

This report was prepared as an account of work sponsored by the United States Government. Neither the United States nor the United States Department of Energy, nor any of their employees, makes any warranty, express or implied, or assumes any legal liability or responsibility for the accuracy, completeness, or usefulness of any information, apparatus, product, or process disclosed, or represents that its use would not infringe privately owned rights. Reference herpin in any sperifir rommorsial produgt, opoocsg. or stirivir liy trado namo. mork, manufocturer, or other wise. Jues nor necessarily constitute or imply its endorsement, recummendation, or favoring by the United States Government or any agency thereot. The views and opinions of authors expressed herein do not necessarily state or reflect those of the United States Governmient or any zoency thereof. 
TABLE OF CONTENTS

Section

Page

INTRODUCTION .......................... ii

I. $\quad$ EXECUTIVE SUMMARY $\ldots \ldots \ldots \ldots \ldots \ldots \ldots \ldots \ldots \ldots \ldots$

II. $\quad$ BATHYMETRY $\ldots \ldots \ldots \ldots \ldots \ldots \ldots \ldots \ldots \ldots \ldots \ldots \ldots \ldots$ II

III. THERMAL RESOURCE................ III-1

IV. MIXED LAYER DEPTH................. IV-I

V. WEATHER CONDITIONS ................ v-1

VI. SEA AND SWELL CHARACTERISTICS........... VI-I

VII. $\quad$ CURRENTS ...................... VII-1

REFERENCES............................. R-1 
INTRODUCTION

One of the basic environmental considerations in site selection for an Ocean Thermal Energy Conversion (OTEC) power plant is the availability of an adequate temperature difference resource. OTEC plants are designed to convert the potential energy in the temperature difference between the warm ocean surface water and the cold water existing at deeper depths into electricity. The turbines which produce the electricity must run on temperature differentials which are extremely small by the standards of conventional energy plants. Therefore, a definition of the most probable temperature structure for a site is most important.

In order to define temperature structures for OTEC areas of interest, Ocean Data Systems, Inc. (ODSI) has developed computer data files of all unclassified soundings available. The primary sources for the data were NOAA's National Oceanographic Data Center, and the U.S. Navy's Fleet Numerical Weather Central. The files were updated in September 1978. Included in the data base were mechanical bathythermographs (MBT), expendable bathythermographs (XBT), salinity temperature depth systems (STD), and Nansen casts.

Under U.S. Department of Energy, Division of Solar Energy Contract No. EY-78-C-2989, ODSI has analyzed the monthly temperature structure for ten different geographical areas. The ten sites selected for study by the Department of Energy are: 


\section{Latitude Longitude}

Bottom Mounted

or

\begin{tabular}{|c|c|c|c|}
\hline Shore Plants: & Dampier Land & $1,3-18^{\circ} \mathrm{S}$ & $118-121^{\circ} \mathrm{E}$ \\
\hline & Manila & $14-16^{\circ} \mathrm{N}$ & $118-120^{\circ} \mathrm{E}$ \\
\hline & Guam & $12-15^{\circ} \mathrm{N}$ & $142-146^{\circ} \mathrm{E}$ \\
\hline & Ivory Coast & $3-6^{\circ} \mathrm{N}$ & $3-8^{\circ} \mathrm{W}$ \\
\hline & Jakarta & $6-9^{\circ} \mathrm{S}$ & $104-109^{\circ} \mathrm{E}$ \\
\hline & Mexico & $20-23^{\circ} \mathrm{N}$ & $105-110^{\circ} \mathrm{W}$ \\
\hline 1.4 & Sri Lanka & $5-10^{\circ} \mathrm{N}$ & $78-83^{\circ} \mathrm{E}$ \\
\hline & Muniliasa & $2 \circ 2-3.4$ & $40-45^{\circ} \mathrm{E}$ \\
\hline Ship Plants: & No. 1 & $5-10^{\circ} \mathrm{N}$ & $90-95^{\circ} \mathrm{W}$ \\
\hline & No. 2 & $13-15^{\circ} \mathrm{N}$ & $75-80^{\circ} \mathrm{W}$ \\
\hline
\end{tabular}

The location of these sites is shown on the following map; a separate report was produced for each site.

For each area, the most probable temperature structures were determined. When the most probable temperature soundings were plotted, some month to month variability was present which was caused by the non-uniform data sample rather than by real changes in the ocean. These short-per1od t1me variations were removed by a filtering process described by Wolff, et al (1977), [44]. Availability óf cold and warm water was examined at each site. In addition to warm and cold water availability, there are other requirements for the continuous operation of an OTEC plant. An adequate temperature differential $(\Delta T)$ is the primary need. A, $\Delta T$ greater than $16.7^{\circ} \mathrm{C}\left(30^{\circ} \mathrm{F}\right)$ for the coldest month of the year would enable year round operation. The annual mean $\Delta T$ for 


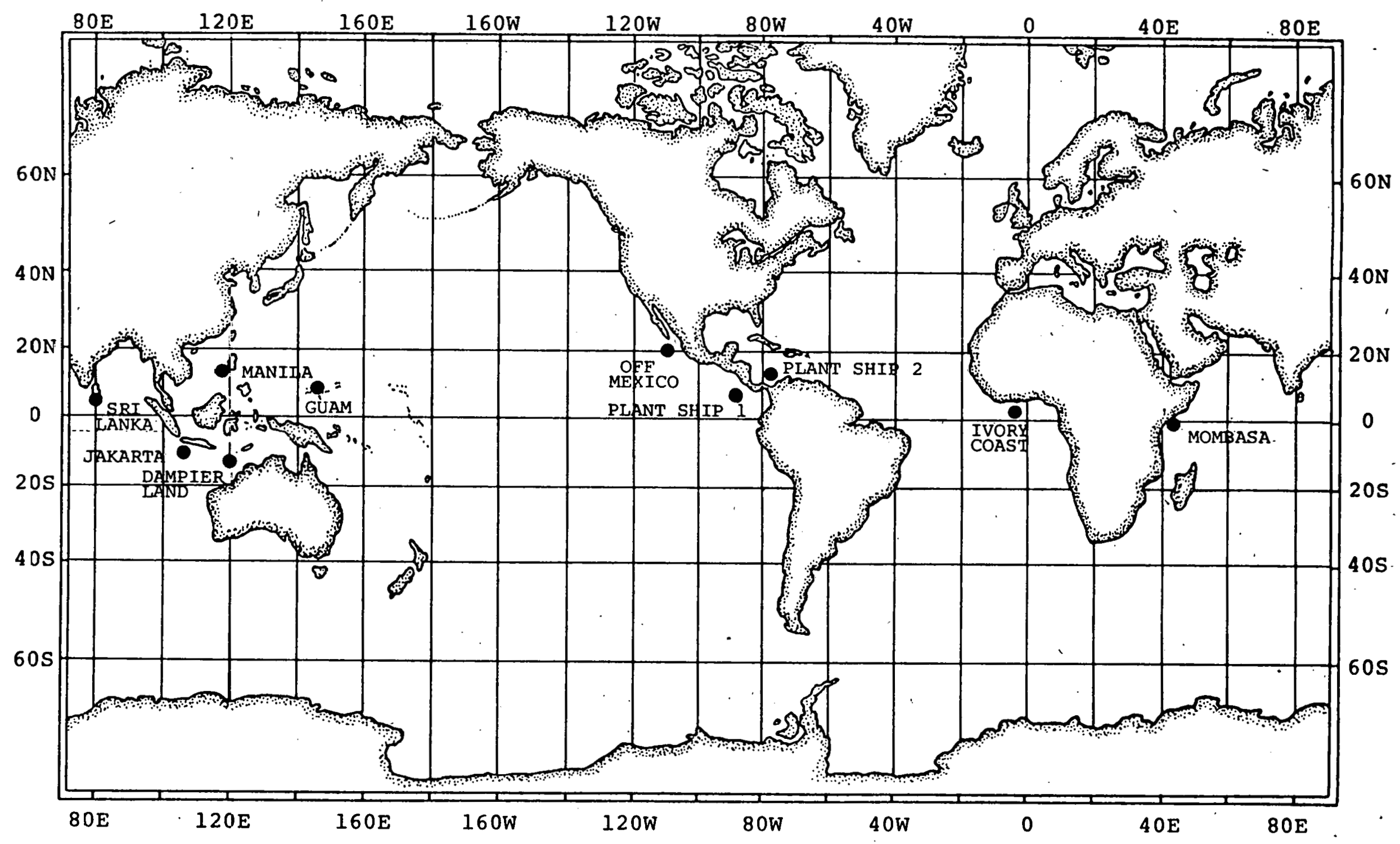

LOCATION OF THE TEN SELECTED SITES 
a site should equal or exceed $20^{\circ} \mathrm{C}\left(36^{\circ} \mathrm{F}\right)$. Besides the thermal resource, there are other operational requirements. Bottom depth should be less than 1500 meters for mooring. Minimum distances offshore to 1000-meter depth is another important consideration for shore plants. Currents should be sufficient to guarantee good cold/warm water sources and to provide for aispersion of modified water. Desirable sites also have light winds, minimum sea and swell, and the lack of severe storms. These parameters have been examined for each site. 'l'he following tables summarize some of the key site parameters for each location. 
SUMMARY OF SITE PARAMETERS

\begin{tabular}{|c|c|c|c|c|c|c|}
\hline Site & \begin{tabular}{|c|} 
Monthly Mean \\
Temperature $\left({ }^{\circ} \mathrm{C}\right)$ \\
Surface Range \\
\end{tabular} & $\begin{array}{c}\text { Monthly Mean } \\
\text { Temperature }\left({ }^{\circ} \mathrm{C}\right) \\
1000 \mathrm{M}\end{array}$ & $\begin{array}{c}\text { Annual } \\
\text { Mean } \\
\Delta \mathrm{T}\left({ }^{\circ} \mathrm{C}\right) 500 \mathrm{M} \\
\end{array}$ & $\begin{array}{c}\text { Annual } \\
\text { Mean } \\
\Delta \mathrm{T}\left({ }^{\circ} \mathrm{C}\right) \quad 1000 \mathrm{M}\end{array}$ & $\begin{array}{c}\text { Coldest Monthly } \\
\text { Mean } \Delta \mathrm{T}\left({ }^{\circ} \mathrm{C}\right) \\
500 \mathrm{M} \\
\end{array}$ & $\begin{array}{c}\text { Coldest Monthly } \\
\text { Mean } \Delta \mathrm{T}\left({ }^{\circ} \mathrm{C}\right) \\
1000 \mathrm{M} \\
\end{array}$ \\
\hline Sri Lanka & $27.5-28.6$ & $6.5-6.7$ & 18.0 & 21.3 & 17.5 & 20.8 \\
\hline Mombasa $\mathrm{N}$ & $25.4-28.7$ & $7.2-7.5$ & 17.0 & 19.5 & 15.6 & 18.2 \\
\hline Mombasa S & $25.5-28.4$ & $6.3-7.0$ & 18.0 & 20.2 & 15.9 & 18.5 \\
\hline Jakarta & $27.2-29.0$ & $5.1-5.6$ & 19.1 & 22.8 & 17.9 & 21.9 \\
\hline Dampier Land & $25.6-28.2$ & $4.9-5.0$ & 19.1 & 22.6 & 17.4 & 20.7 \\
\hline Manila & $27.1-29.5$ & $4.4-4.6$ & 20.0 & 24.0 & 18.6 & 22.6 \\
\hline Guam & $27.7-29.2$ & $4.3-4.4$ & 21.1 & 24.1 & $20: 4$ & 23.4 \\
\hline off Mexico & $22.5-28.0$ & $4.4-4.5$ & 17.6 & 20.9 & 14.9 & 18.0 \\
\hline $\begin{array}{c}\text { Plant Ship } \\
\text { Pacific }\end{array}$ & $27.1-28.5$ & $4.6-4.8$ & 19.4 & 22.8 & .18 .1 & 21.7 \\
\hline Ivory Coast & $24.3-28.1$ & 4.5 & 19.2 & 22.1 & 16.8 & 19.7 \\
\hline $\begin{array}{l}\text { Plant Ship } \\
\text { Caribbean }\end{array}$ & $26.4-28.4$ & $5.0-5.3$ & 18.1 & 22.4 & 17.2 & 21.3 \\
\hline
\end{tabular}


SUMMARY OF SITE PARAMETERS

\begin{tabular}{|c|c|c|c|c|c|}
\hline SITE & $\begin{array}{l}\text { RANGE DISTANCE } \\
\text { (IN KILOMETERS) } \\
\text { TO SHORE FROM } \\
\text { 1000 METERS } \\
\end{array}$ & $\begin{array}{l}\text { MONTHEY MEAN } \\
\text { MIXED LAYER } \\
\text { DEPTH METERS }\end{array}$ & $\begin{array}{l}\text { MONTHLY MEAN } \\
\text { SURFACE CURRENTS } \\
\text { (CM/SEC) }\end{array}$ & $\begin{array}{l}\text { SEA STATE MAX } \\
\& \text { OF TIME }>3 \\
\text { METE RS }\end{array}$ & $\begin{array}{l}\text { NUMBER TROPICAL } \\
\text { CYCLONES PER } \\
\text { YEAR }\end{array}$ \\
\hline SRI LANKA & $22-55$ & $30-80$ & $25-62$ & 3 & $0.2-1.2$ \\
\hline MOMBASA & $33-130$ & $30-90$ & $30-62$ & 2 & $0.0-0.1$ \\
\hline JAKARTA & $18-60$ & $55-80$ & $25-52$ & 2 & $0.0-0.1$ \\
\hline DAMPIER LAND & $265-417$ & $30-80$ & $25-47$ & 4 & $0.4-1.2$ \\
\hline MANILA & $6-82$ & $20-80$ & $30-52$ & & $4.0-6.0$ \\
\hline GUAM & $7-18$ & $60-120$ & $30-47$ & 5 & $2.0-3.0$ \\
\hline OFF MEXICO & $5-104$ & $10-30$ & $25-31$ & 2 & $0.6-4.0$ \\
\hline $\begin{array}{l}\text { PLANT SHIP } \\
\text { PACIFIC }\end{array}$ & - & $0-30$ & $30-52$ & 2 & $0.0-2.0$ \\
\hline IVORY COAST & $33-52$ & $0-30$ & $25-31$ & 2 & $0.0-0.1$ \\
\hline $\begin{array}{l}\text { PLANT SHIP } \\
\text { CARIBBEAN }\end{array}$ & - & $40-110$ & $30-62$ & 3 & $0.6-1.2$ \\
\hline
\end{tabular}


I. EXECUTIVE SUMMARY

The region off Dampier Land, Australia was selected for study as a potential OTEC site. The region examined was between $13-18^{\circ}$ South latitude and 118-121 East longitude. Data coverage was sparse, but the existing data demonstrated a fine potential for Ocean Thermal Energy Conversion (OTEC) use. The maximum temperature gradient remains relatively stable throughout the year. The annual average $\Delta \mathrm{T}$ at 1000 meters is $22.6^{\circ} \mathrm{C}$. At 600 meters, an annual average $\Delta \mathrm{T}$ greater than $20^{\circ} \mathrm{C}$ is obtainable.

The continental shelf off Dampier Land is very wide; thus, the distance to water 1500 meters deep may be as much as 480 kilometers (260 nautical miles). Water 1000 meters deep lies at least 265 kilometers (143 nautical miles) offshore. This distance is a major problem, perhaps necessitating the use of a plant ship. Tropical storms can be a problem between December and April. The ocean off Dampier Land has an upper mixed layer throughout the year. Currents are generally moderate, although the surface circulation pattern changes during the year. 
II : BATHYMETRY.

The location of the area off Dampier Land is shown on Figure II-1. Figure II-2 shows the rough bathymetry off the Dampier Land coast taken from Defense Mapping Agency Hydrographic Center (1974), [10]. The continental shelf in this area is relatively wide with long distances from acceptable depths to an installation on shore. Table II-l summarizes distances to selected depths off Dampier Land.

TABLE II-1: DISTANCES TO SELECTED DEPTHS OFF DAMPIER LAND. From Defense Mapping Agency (1974), [10].

\begin{tabular}{|c|c|c|}
\hline $\begin{array}{c}\text { DEPTH } \\
\text { (Meters) }\end{array}$ & $\begin{array}{c}\text { CLOSEST DISTANCE } \\
\text { (Kilometers) }\end{array}$ & $\begin{array}{c}\text { FARTHEST DISTANCE } \\
\text { (Kilometers) }\end{array}$ \\
\hline 100 & 98 & $28-3$ \\
500 & 218 & 408 \\
1000 & 265 & 417 \\
1500 & 293 & 482 \\
\hline
\end{tabular}

Because of the long distances to shore, a floating OTEC plant should be considered for this area. 


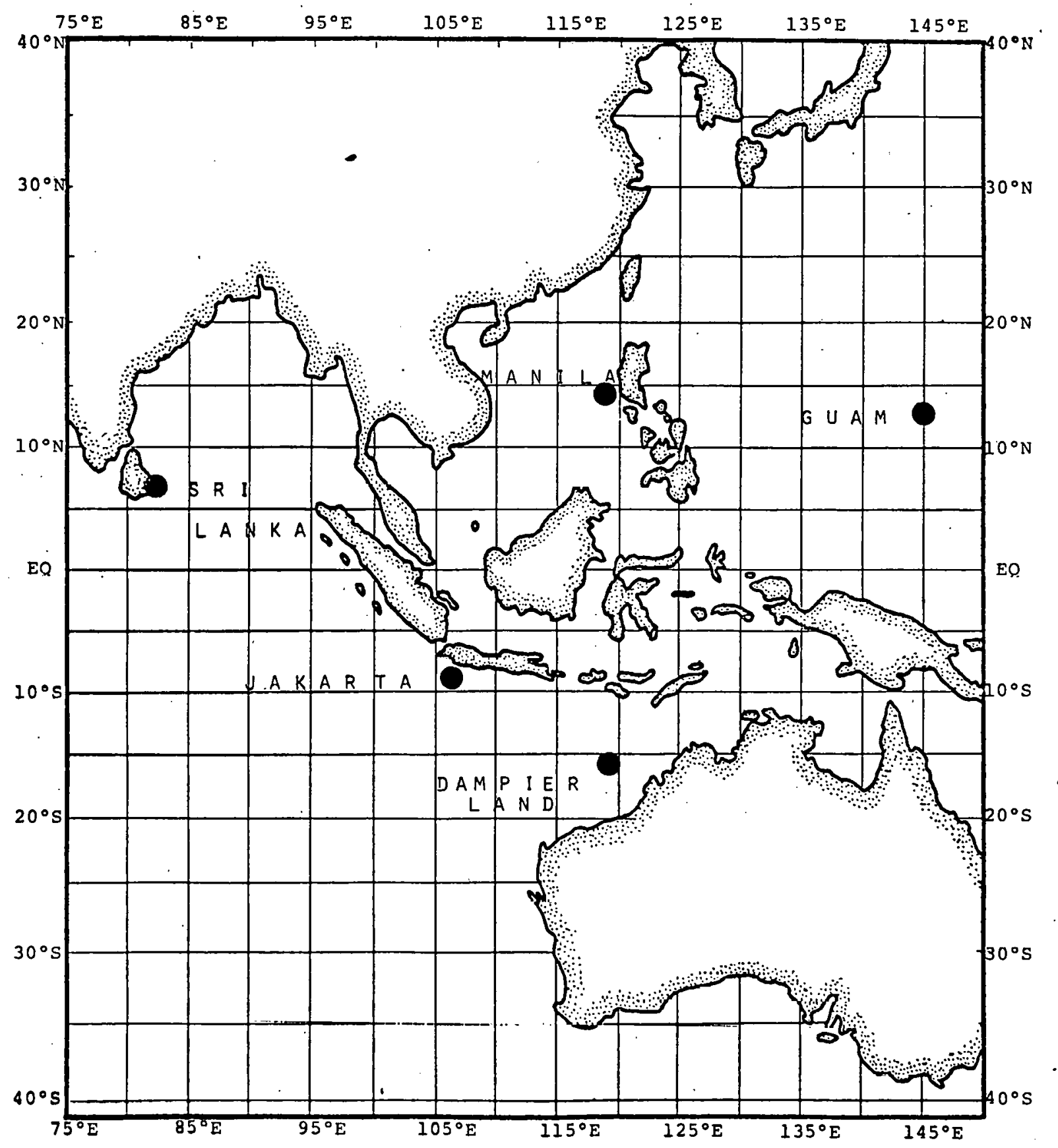

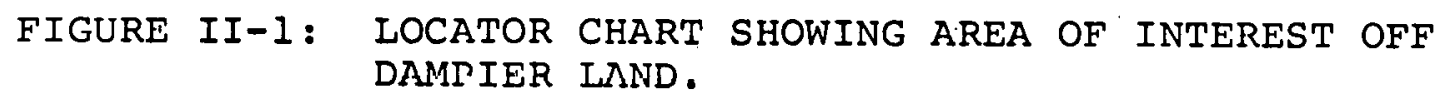

I I - 2 


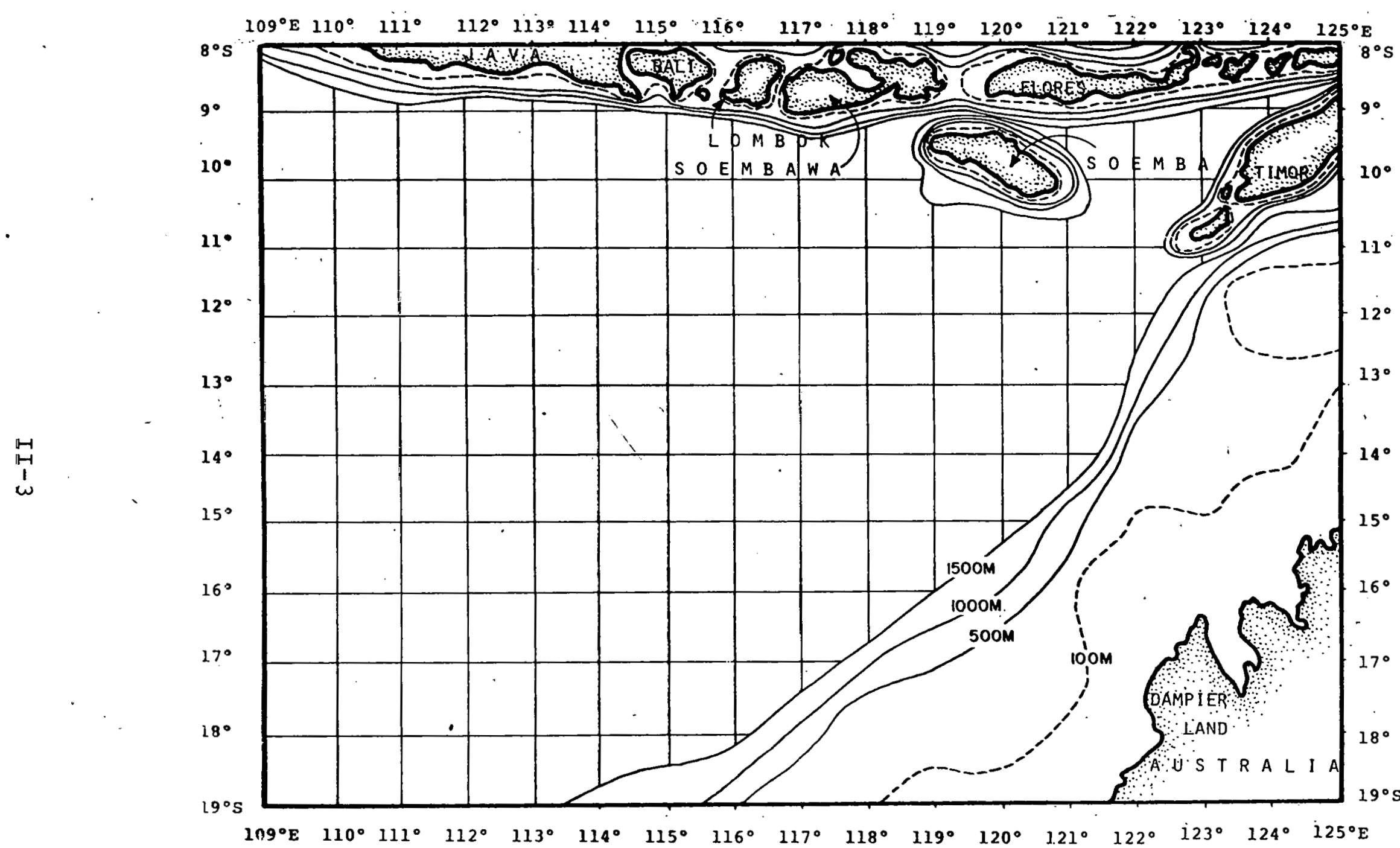

IIGUIE II-2: CHART SHOWING ROUGH BATHYMETRY OFF DAMPIER LAND. FrOm Defense Mapping Agency (1974) [피] . 
III. THERMAL RESOURCE

Even through a large area was selected for study, there was a paucity of sounding data during some months and at some depths. The ODSI master subsurface file was the main source for evaluation of the thermal resource; however, Colburn (1974):[6] and wyrtki (1971) [47]. were used as additional references.

The data available indicate a thermal structure with good potential for OTEC operations. An annual average $\triangle T$ of greater than $20^{\circ} \mathrm{C}$ is available at a depth of 600 meters. The area has a permanent, broad thermocline during all months. There is also an ample supply of warm surface water with mean temperatures ranging from 25.6 to $28.2^{\circ} \mathrm{C}$. Open access to Antarctic source regions assures a good cold water. supply.

Table III-l shows the monthly most probably temperature structure off Dampier Land as a function of depth. Table III-2 shows the same data presented as $\Delta T$, (surface temperature minus temperature at depth) while Figure III-l shows monthly $\Delta \mathrm{T}$ contours for the area off Dampier Land. Short period fluctuations due to the sparse data sample were removed using a filtering process described by wolff, et al $(1977),[44]$. 
TABLE III-1: MONTHLY. MOST PROBABLE TEMPERATURE ( $\left.{ }^{\circ} \mathrm{C}\right)$ PROFILE DAMPIER LAND $13-18^{\circ} \mathrm{S} / 118-121^{\circ} \mathrm{E}$

\begin{tabular}{|c|c|c|c|c|c|c|c|c|c|c|c|c|c|}
\hline Depth & 1 & 2. & 3 & 4 & 5 & $\begin{array}{c}\text { Month } \\
6\end{array}$ & 7 & 8 & 9 & 10 & 11 & 12 & $\begin{array}{c}\text { Annual } \\
\text { Mean }\end{array}$ \\
\hline 0 & 28.1 & 27.5 & 27.3 & 27.8 & 27.5 & 26.8 & 25.6 & 25.6 & 26.1 & 27.4 & 28.2 & 28.2 & 27.2 \\
\hline 50 & 26.5 & 26.9 & 26.3 & 27.0 & 27.1 & 26.6 & 25.2 & 25.2 & 25.3 & 25.9 & 26.2 & 26.7 & 26.2 \\
\hline 100 & 23.4 & 22.9 & 22.8 & 23.5 & 24.5 & 25.0 & 24.2 & 24.1 & 24.1 & 24.1 & 23.9 & 23.5 & 23.8 \\
\hline 150 & 19.8 & 19.6 & 19.9 & 20.3 & 20.8 & 21.7 & 21.3 & 21.4 & 20.9 & 21.1 & 20.7 & 20.0 & 20.6 \\
\hline 200 & 15.6 & 15.6 & 15.6 & 16.2 & 16.7 & 17.5 & 17.3 & 17.2 & .16 .9 & 17.1 & 17.0 & 16.5 & 16.6 \\
\hline 250 & 13.2 & 12.9 & 12.8 & 13.0 & 13.3 & 14.1 & 14.1 & 14.3 & 14.3 & 13.9 & 14.1 & 13.7 & 13.6 \\
\hline 300 & 11.3 & 10.9 & 10.8 & 11.1 & 11.5 & 11.9 & 12.0 & 12.0 & 11.8 & 11.8 & 11.8 & 11.6 & 11.5 \\
\hline 350 & 10.1 & 10.0 & 10.2 & 10.3 & 10.4 & 10.5 & 10.6 & 10.6 & 10.6 & 10.4 & 10.5 & 10.3 & 10.4 \\
\hline 400 & 9.4 & 9.3 & 9.3 & 9.5 & 9.5 & 9.6 & 9.6 & 9.6 & 9.6 & 9.5 & 9.5 & 9.4 & 9.5 \\
\hline 450 & 8.6 & 8.6 & 8.6 & 8.7 & 8.8 & 8.9 & 8.9 & 8.8 & 8.8 & 8.7 & 8.7 & 8.6 & 8.7 \\
\hline 500 & 8.1 & 8.1 & 8.1 & 8.2 & 8.3 & 8.3 & 8.2 & 8.1 & 8.1 & 8.0 & 8.0 & 8.0 & 8.1 \\
\hline 550 & 7.6 & 7.6 & 7.6 & 7.7 & 7.8 & 7.8 & 7.7 & 7.5 & 7.5 & 7.4 & 7.5 & 7.5 & 7.6 \\
\hline $600^{\circ}$ & 7.1 & 7.1 & 7.2 & 7.3 & 7.3 & 7.3 & 7.2 & 7.1 & 7.0 & 6.9 & 7.0 & 7.0 & 7.2 \\
\hline 650 & 6.7 & 6.7 & 6.8 & 6.9 & 6.9 & 6.9 & 6.8 & 6.7 & 6.6 & 6.6 & 6.6 & 6.6 & 6.7 \\
\hline 700 & 6.4 & 6.4 & 6.4 & 6.5 & 6.5 & 6.4 & 6.3 & 6.3 & 6.3 & 6.2 & 6.3 & 6.3 & 6.4 \\
\hline 750 & 6.1 & 6.0 & 6.0 & 6.1 & 6.1 & 6.1 & 6.0 & 6.0 & 6.0 & 6.0 & 6.0 & 6.0 & 6.0 \\
\hline 800 & 5.8 & 5.8 & 5.8 & 5.8 & 5.9 & $5: 8$ & 5.8 & 5.8 & 5.8 & 5.7 & 5.7 & 5.8 & 5.8 \\
\hline 850 & 5.6 & 5.5 & 5.5 & $5: 5$ & 5.6 & 5.5 & 5.5 & 5.5 & 5.5 & 5.4 & 5.5 & 5.6 & 5.5 \\
\hline 900 & 5.4 & 5.4 & 5.3 & 5,4 & 5.4 & 5.4 & 5.3 & 5.3 & 5.3 & 5.3 & 5.3 & 5.4 & 5.3 \\
\hline 950 & 5.2 & 5.1 & 5.1 & 5.1 & 5.1 & 5.1 & 5.1 & 5.1 & 5.1 & 5.1 & 5.1 & 5.1 & 5.1 \\
\hline 1000 & 5.0 & $5: 0$ & 4.9 & 4.9 & 4.9 & 4.9 & 4.9 & 4.9 & 4.9 & 4.9 & 4.9 & 5.0 & 4.9 \\
\hline 1050 & 4.8 & 4.8 & 4.7 & 4.8 & 4.8 & 4.8 & 4.7 & 4.6 & 4.7 & 4.7 & 4.7 & 4.8 & 4.7 \\
\hline 1100 & 4.6 & 4.6 & 4.6 & 4.6 & 4.6 & 4.5 & 4.5 & 4.5 & 4.5 & 4.6 & 4.6 & 4.6 & 4.6 \\
\hline 1150 & 4.5 & 4.4 & 4.4 & 4.4 & 4.4 & 4.4 & 4.4 & 4.4 & 4.4 & 4.4 & 4.5 & 4.5 & 4.4 \\
\hline 1200 & 4.3 & 4.3 & 4.3 & 4.3 & 4.3 & 4.2 & 4.2 & 4.2 & 4.2 & 4.2 & 4.3 & 4.3 & 4.3 \\
\hline 1250 & 4.2 & 4.2 & 4.1 & 4.1 & 4.1 & 4.1 & 4.1 & 4.1 & 4.1 & 4.1 & 4.2 & 4.2 & 4.1 \\
\hline $13 n n$ & 4.1 & 4.0 & $4, n$ & 4.0 & 4.0 & 4.0 & 4,0 & 3.9 & 3,9 & 3,9 & 4.0 & 4.1 & 4,0 \\
\hline 1350 & 3.9 & 3.8 & 3.8 & 3.8 & 3.8 & 3.8 & 3.8 & 3.8 & 3.8 & 3.8 & 3.9 & 3.9 & 3.8 \\
\hline 1400 & 3.7 & 3.7 & 3.7 & 3.7 & 3.6 & 3.6 & 3.7 & 3.7 & 3.7 & 3.7 & 3.7 & 3.7 & 3.7 \\
\hline 1450 & 3.5 & 3.5 & 3.6 & 3.6 & 3.5 & 3.5 & 3.5 & 3.6 & 3.6 & 3.6 & 3.6 & 3.5 & 3.6 \\
\hline 1500 & 3.4 & 3.4 & 3.4 & 3.4 & 3.4 & 3.4 & 3.4 & 3.4 & 3.5 & 3.5 & 3.4 & 3.4 & 3.4 \\
\hline
\end{tabular}


TABLE III-2: SURFACE TEMPERATURE - TEMPERATURE AT DEPTH DIFFERENTIAL $\left({ }^{\circ} \mathrm{C}\right)$ BY MONTHS DAMPIER LAND $13-18^{\circ} \mathrm{S} / 118-121^{\circ} \mathrm{E}$

\begin{tabular}{|c|c|c|c|c|c|c|c|c|c|c|c|c|c|}
\hline \multirow[t]{2}{*}{ Depth } & \multicolumn{11}{|c|}{ Month } & \multirow{2}{*}{\multicolumn{2}{|c|}{$\begin{array}{c}\text { Annual } \\
\text { Mean }\end{array}$}} \\
\hline & 1 & 2 & 3 & 4 & 5 & 6 & 7 & 8 & 9 & $10^{\circ}$ & 11 & & \\
\hline 50 & 1.6 & 0.6 & 1.0 & 0.8 & 0.4 & 0.2 & 0.4 & 0.4 & 0.8 & 1.5 & 2.0 & 1.5 & 0.9 \\
\hline 100 & 4.7 & 4.6 & 4.5 & 4.3 & 3.0 & 1.8 & 1.4 & 1.5 & 2.0 & 3.3 & 4.3 & 4.7 & 3.3 \\
\hline 150 & 8.3 & 7.9 & 7.4 & 7.5 & 6.7 & 5.1 & 4.3 & 4.2 & 5.2 & 6.3 & 7.5 & 8.2 & 6.6 \\
\hline 200 & 12.5 & 11.9 & 11.7 & 11.6 & 10.8 & 9.3 & 8.3 & 8.4 & 9.2 & 10.3 & $11.2^{\circ}$ & 11.7 & 10.6 \\
\hline 250 & 14.9 & 14.6 & 14.5 & 14.8 & 14.2 & 12.7 & 11.5 & 11.3 & 11.8 & 13.5 & 14.1 & 14.5 & 13.5 \\
\hline 300 & 16.8 & 16.6 & 16.5 & 16.7 & 16.0 & 14.9 & 13.6 & 13.6: & 14.3 & 15.6 & 16.4 & 16.6 & 15.6 \\
\hline 350 & 18.0 & 17.5 & 17.1 & 17.5 & 17.1 & 16.3 & 15.0 & 15.0 & 15.5 & 17.0 & 17.7 & 17.9 & 16.8 \\
\hline 400 & 18.7 & 18.2 & 18.0 & 18.3 & 18.0 & 17.2 & 16.0 & 16.0 & 16.5 & 17.9 & 18.7 & 18.8 & $17 ! 7$ \\
\hline 450 & 19.5 & 18.9 & 18.7 & 19.1 & 18.7 & 17.9 & 16.7 & 16.8 & 17.3 & 18.7 & 19.5 & 19.6 & 18.5 \\
\hline 500 & 20.0 & 19.4 & 19.2 & 19.6 & 19.2 & 18.5 & 17.4 & 17.5 & 18.0 & 19.4 & 20.2 & 20.2 & 19.1 \\
\hline 550 & 20.5 & 19.9 & 19.7 & 20.1 & 19.7 & 19.0 & 17.9 & 18.1 & 18.6 & 20.0 & 20.7 & 20.2 & 19.6 \\
\hline 600 & 21.0 & 20.4 & 20.1 & 20.5 & 20.2 & 19.5 & 18.4 & 18.5 & 19.1 & 20.5 & 21.2 & 21.2 & 20.1 \\
\hline 650 & 21.4 & 20.8 & 20.5 & 20.9 & 20.6 & 19.9 & 18.8 & 18.9 & 19.5 & 20.8 & 21.6 & 21.6 & 20.4 \\
\hline 700 & 21.7 & 21.1 & 20.9 & 21.3 & 21.0 & 20.4 & 19.3 & 19.3 & 19.8 & 21.2 & 21.9 & 21.9 & 20.8 \\
\hline 750 & 22.0 & 21.5 & 21.3 & 21.7 & 21.4 & 20.7 & 19.6 & 19.6 & 20.1 & 21.4 & 22.2 & 22.2 & 21.1 \\
\hline 800 & 22.3 & 21.7 & 21.5 & 22.0 & 21.6 & 21.0 & 19.8 & 19.8 & 20.3 & 21.7 & 22.5 & 22.4 & 21.4 \\
\hline 850 & 22.5 & 22.0 & 21.8 & 22.3 & 21.9 & 21.3 & 20.1 & 20.1 & 20.6 & 22.0 & 22.7 & 22.6 & 2.1 .7 \\
\hline 900 & 22.7 & 22.1 & 22.0 & 22.4 & 22.1 & 21.4 & 20.3 & 20.3 & 20.8 & 22.1 & $22.9^{\circ}$ & 22.8 & 21.8 \\
\hline 950 & 22.9 & 22.4 & 22.2 & 22.7 & 22.4 & 21.7 & 20.5 & 20.5 & 21.0 & 22.3 & 23.1 & 23.1 & 22.1 \\
\hline 1000 & 23.1 & 22.5 & 22.4 & 22.9 & 22.6 & 21.9 & 20.7 & 20.7 & 21.2 & 22.5 & 23.3 & 23.2 & 22.6 \\
\hline 1050 & 23.3 & 22.7 & 22.6 & 23.0 & 22.7 & 22.0 & 20.9 & 21.0 & 21.4 & 22.7 & 23.5 & 23.4 & 22.4 \\
\hline 1100 & 23.5 & 22.9 & 22.7 & 23.2 & 22.9 & 22.3 & 21.1 & 21.1 & 21.6 & 22.8 & 23.6 & 23.6 & 22.6 \\
\hline 1150 & 23.6 & 23.1 & 22.9 & 23.4 & 23.1 & 22.4 & 21.2 & 21.2 & 21.7 & 23.0 & 23.7 & 23.7 & 22.8 \\
\hline 1200 & 23.8 & 23.2 & 23.0 & 23.5 & 23.2 & 22.6 & 21.4 & 21.4 & 21.9 & 23.2 & 23.9 & 23.9 & 22.9 \\
\hline 1250 & 23.9 & 23.3 & 23.2 & 23.7 & 23.4 & 22.7 & 21.5 & 21.5 & 22.0 & 23.3 & 24.0 & 24.0 & 23.0 \\
\hline 1300 & 24.0 & 23.5 & $23 . .3$ & 23.8 & 23.5 & 22.8 & 21.6 & 21.7 & 22.2 & 23.5 & 24.2 & 24.1 & 23.2 \\
\hline 1350 & 24.2 & 23.7 & 23.5 & 24.0 & 23.7 & 23.0 & 21.8 & 21.8 & 22.3 & 23.6 & 24.3 & 24.3 & 23.3 \\
\hline 1400 & 24.4 & 23.8 & 23.6 & 24.1 & 23.9 & 23.2 & 21.9 & 21.9 & 22.4 & 23.7 & 24.5 & 24.5 & 23.5 . \\
\hline 1450 & 24.6 & 24.0 & 23.7 & 24.2 & 24.0 & 23.3 & 22.1 & 22.0 & 22.5 & 23.8 & 24.6 & 24.7 & 23.6 \\
\hline 1500 & 24.7 & 24.1 & 23.9 & 24.4 & 24.1 & 23.4 & 22.2 & 22.2 & 22.6 & 23.9 & 24.8 & 24.8 & 23.8 \\
\hline
\end{tabular}




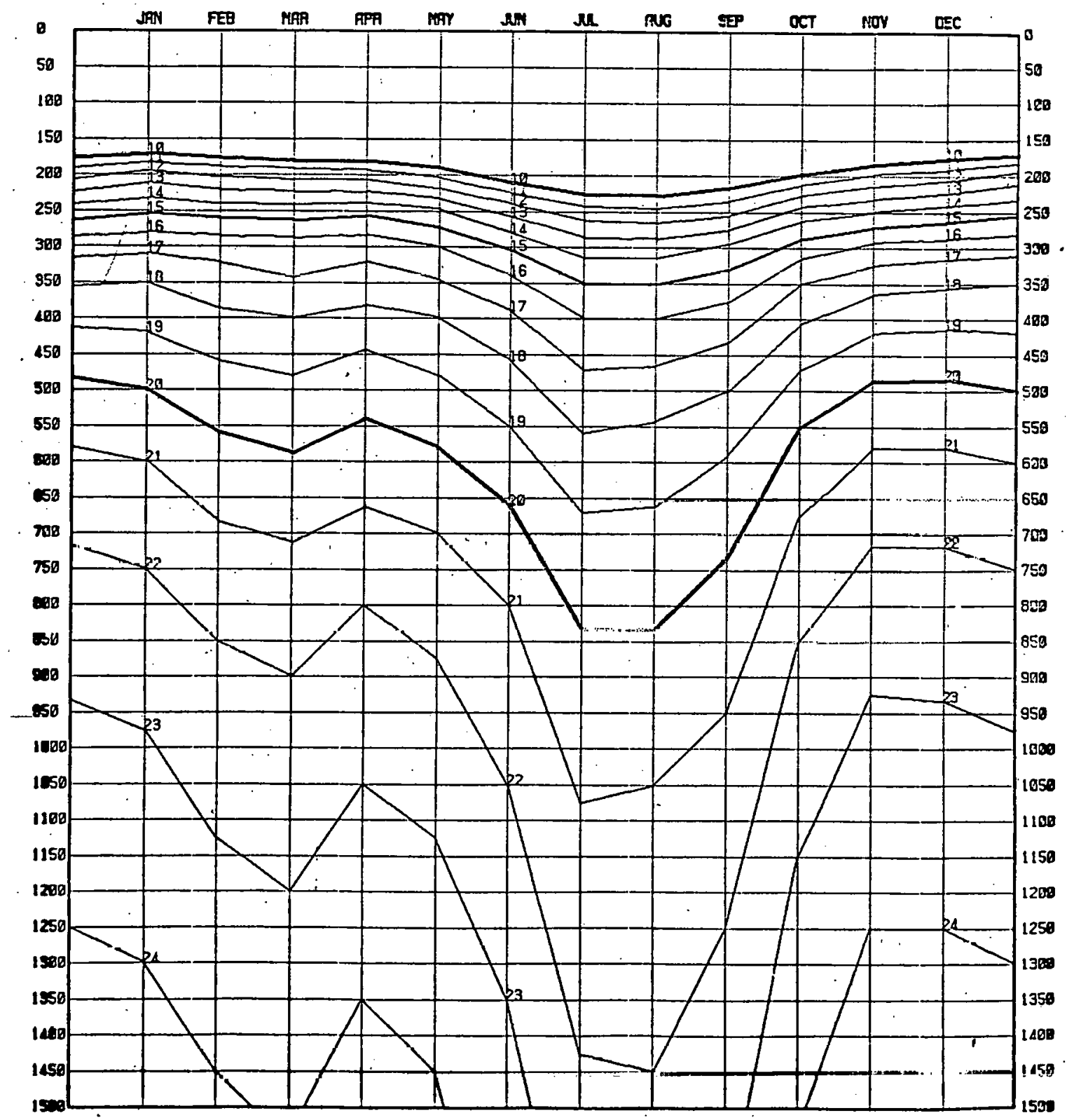

FIGURE III-1: CHART SHOWING MONTHLY $\triangle T$ CONTOURS $\left({ }^{\circ} \mathrm{C}\right)$ AT 50-METER DEPTH INTERVALS FOR THE AREA OFF DAMPIER LAND, AUSTRALIA (13-18S, 118-121E). 
IV. MIXED LAYER DEPTH

An adequate mixed layer depth (MLD) exists the year round. The MLD is deep enough to assure an intake of uniformly warm water, and shallow enough so that the mixed exhaust water can be discharged below the MLD in order to minimize the possibility of recirculation. Because of the distance offshore, a floating OTEC plant was recommended for consideration in this area; this would further enhance the safe dispersion of modified waters.

Mixed layer depths in this area were determined from the profiles used in the thermal resource assessment (section III) and the investigations of Colburn (1974), [무]. TypicaI monthly MLD values are shown' in Table'IV-l.

TABLE IV-I: TYPICAL MIXED LAYER DEPTHS (METERS) OFF DAMPIER LAND; AUSTRALIA.

$\begin{array}{llllll}\frac{\text { JAN }}{40} & \frac{\text { FEB }}{30} & \frac{\text { MAR }}{30} & \frac{\text { APR }}{40} & \frac{M A Y}{50} & \frac{\text { JUN }}{70} \\ \frac{\text { JUL }}{80} & \frac{\text { AUG }}{80} & \frac{\text { SEP }}{70} & \frac{\text { OCT }}{60} & \frac{\text { NOV }}{40} & \frac{\text { DEC }}{40}\end{array}$


V. WINDS AND STORMS

The proposed site lies within the climatological region of the Southeast Trade Winds, and storms are a potential problem for OTEC operations off Dampier Land. The main season for storms is from December to April, with significant occurrences in May, June, and November. The monthly average tropical storms per year are as follows:

JAN FEB MAR APR MAY JUN JUL AUG SEP OCT NOV DEC

Tropical Storms

$\begin{array}{llllllllllll}3 & 4 & 5 & 1 & 0 & 0 & 0 & 0 & 0 & 0 & 0 & 2\end{array}$

This data was derived from H.L. Crutcher and R.G. Quàyle (1974), [] $]$ Figure $V-1$ and $V-2$ are adapted from the same source. Figure $\mathrm{V}-1$ shows the preferred annual storm tracks for the region. Figure V-2 from the same source provides the average number of tropical cyclones per $5^{\circ}$ square per year. There is a wide range of numbers of tropical storms per year depending upon where in the site region the plant was located. In the northern part, plants would be less affected by tropical storms.

Johnson and Denwick in an NDBO study (1978), [20], shows that a hurricane can cause a temperature anomaly of $-3^{\circ} \mathrm{C}$ for about a four day period. While this result was measured in thé Gulf of. Mexico, the resulting temperature difference and the recovery process is uniform throughout the tropics. Currents will be formed. Mountainous wave 
heights of 10 to 15 meters have been reported on numerous occasions in hurricanes, according to Gentry, (1970), [18]. Sustained wind speeds may approach 50 meters per second. Tables V-1 through V-4, taken from the U.S. Naval Weather Service (1938), [31], shows basic information on the winds for each of the ten sites examined under this contract. 


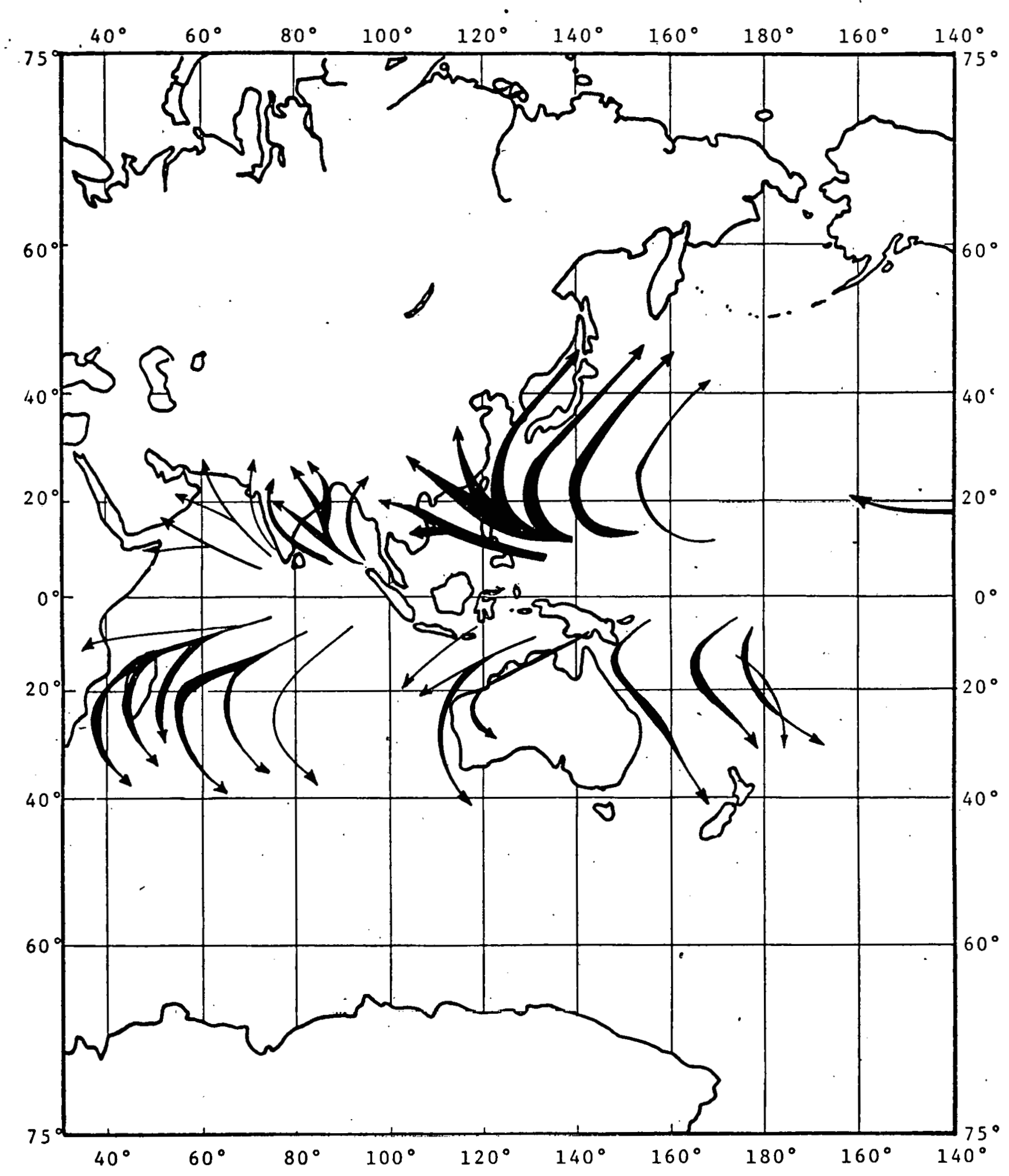

ANNUAL PERFERRED STORM TRACKS FOR TROPICAL STORMS

PIGURE V-I

$\mathrm{V}-3$. 


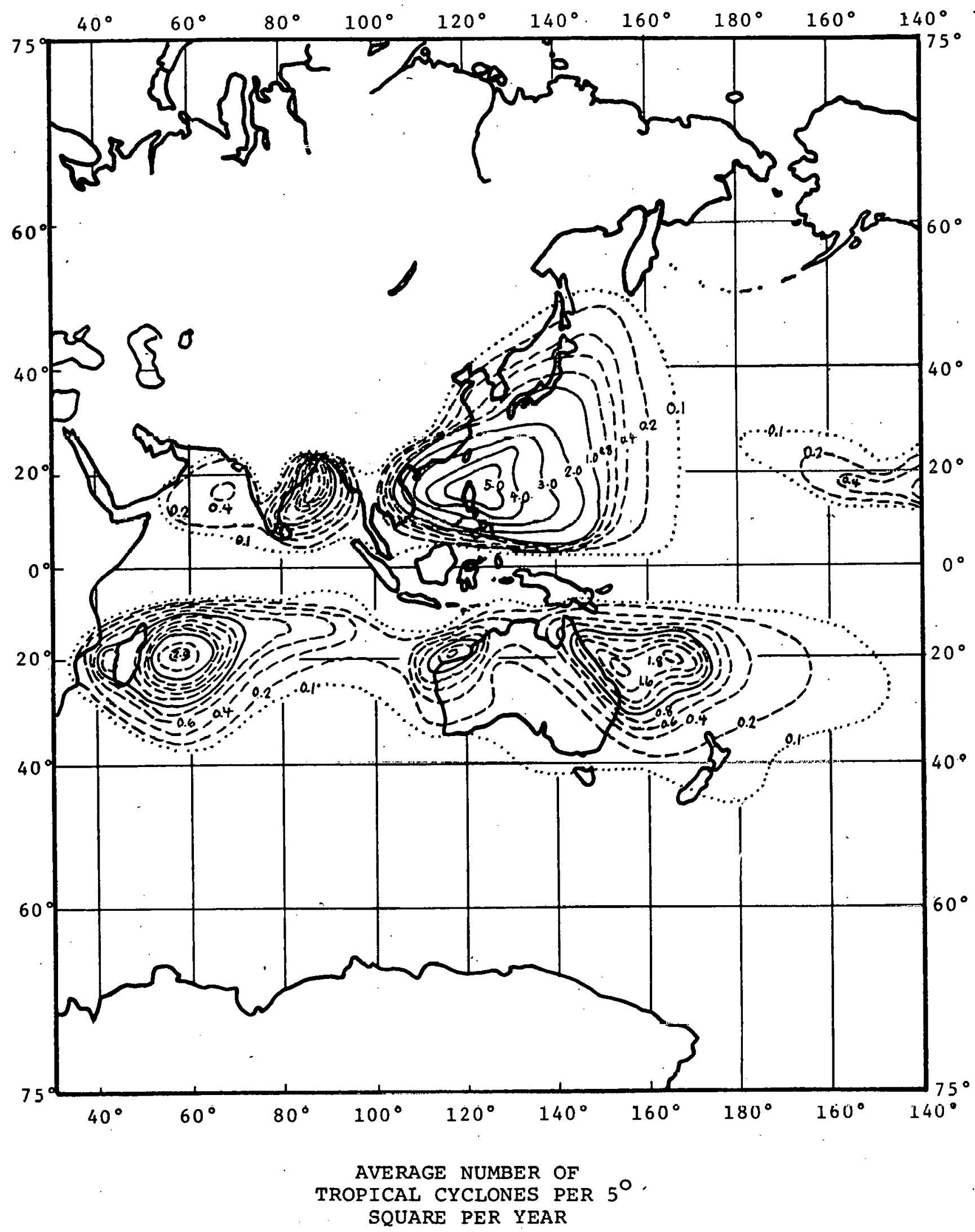

FIGURE V-2

$\mathrm{V}-4$ 
TABLE $\mathrm{V}-1$

RESULTANT WINDS

Average' Wind Velocity in Meters per second

\begin{tabular}{|c|c|c|c|c|}
\hline Location & Dec-Jan-Feb & Mar-Apr-May & Jun-Jul-Aug & Sep-Oct-Nov \\
\hline Ivory Coast & $3.1-\dot{4} .1$ & $3.1-4.1$ & $4.1-5.1$ & $4.1-5.1$ \\
\hline Mombasa & $5.1-6.2$ & $4.1-5.1$ & $6.2-7.2$ & $4.1-5.1$ \\
\hline Sri Lanka & $\begin{array}{l}4.1-5.1 W \\
5.1-6.2 E\end{array}$ & $4.1-5.1$ & $\begin{array}{l}6.2-7.2 \mathrm{~W} \\
7.2-8.2 \mathrm{E}\end{array}$ & $5.1-6.2$ \\
\hline Jakarta & 4.1 & $3.1-4.1$ & $5.1-6.2$ & $5.1-6.2$ \\
\hline Dampier Land & $4.1-5.1$ & $4.1-6.2$ & $5.1-6.2$ & $4.1-5.1$ \\
\hline Philippines & $5.1-6.2$ & $3.1-4.1$ & 4.1 & $6.2-7.2$ \\
\hline Guam & $5.1-6.2$ & $4.1-5.1$ & $4.1-5.1$ & $4.1-5.1$ \\
\hline Off Mexico & $3.1-4.1$ & $2.1-4.1$ & $2.1-4.1$ & $4.1-5.1$ \\
\hline $\begin{array}{l}\text { Plant Ship } \\
\text { Pacific }\end{array}$ & $3.1-4.1$ & $3.1-4 \cdot 1$ & $4.1-5.1$ & $4.1-6.2$ \\
\hline $\begin{array}{l}\text { Plant Ship } \\
\text { Caribbean }\end{array}$ & $6.2-7.2$ & $5.1-6.2$ & $6.2-8.2$ & $4.1-5.1$ \\
\hline
\end{tabular}


TABLE V-2

FREQUENCY AND PERCENTAGE OF MODERATE GALES AND STRONGER WINDS,

BEAUFORT FORCE 7 AND HIGHER

( $\geq 14.4$ meters per second)

\begin{tabular}{|c|c|c|c|c|c|c|c|c|c|c|}
\hline MONTII & $\begin{array}{l}\text { IVORY } \\
\text { COAST }\end{array}$ & MOIBBASA & $\begin{array}{r}\text { SRI } \\
\text { AINKA } \\
\end{array}$ & JAKARTA & $\begin{array}{c}\text { DANPIER } \\
\text { IAND } \\
\end{array}$ & PHIIUIPINES & GUAM & $\begin{array}{c}\text { OFF } \\
\text { MEXICO } \\
\end{array}$ & $\begin{array}{c}\text { PLANT } \\
\text { SHIP } \\
\text { (PACIFIC) }\end{array}$ & $\begin{array}{c}\text { PLANT } \\
\text { SHIP } \\
\text { (CARIBN) }\end{array}$ \\
\hline JAK & $0=$ & $0-1$ & 1) & 0 & 0 & $1-5$ & $1-5$ & $1-5$ & 0 & 5 \\
\hline FEE & 0 & 0 & 1 & $1-5$ & $1-5$ & 5 & $1-2$ & $1-2$ & 0 & 5 \\
\hline MAT. & $1-2$ & 0 & D) & $1-2$ & $1-2$ & 1 & 0 & $1-2$ & 0 & $1-5$ \\
\hline$\triangle P F$ & 0 & 0 & 1) & $1-2$ & 0 & 1 & $1-5$ & 1 & 0 & $1-5$ \\
\hline MAY & 0 & 1 & $1-5$ & 1 & 0 & $1-5$ & 0 & 0 & 0 & 1 \\
\hline JUN & 0 & $5-10$ & $5-10$ & $1-5$ & 0 & $.1-5$ & 0 & 1 & 0 & $1-5$ \\
\hline JUL & 0 & $\begin{array}{r}5-10 N \\
10-20 S\end{array}$ & $1-5$ & $1-5$ & $1-5$ & 5 & 0 & 0 & 0 & $1-5$ \\
\hline AUG & 0 & $\begin{array}{l}1-5 S \\
5-15 \mathrm{~N}\end{array}$ & $1-5$ & 0 & 0 & $1-5$ & $1-5$ & $1-5$ & 0 & $1-5$ \\
\hline SEF & 0 & 0 & $1-5$ & $1-5$ & 0 & 5 & $1-5$ & 0 & 0 & 1 \\
\hline OCT & 0 & 0 & $\begin{array}{c}O N \\
1-5 E\end{array}$ & $1-5$ & 0 & $.1-5$ & $1-2$ & 1 & 1 & $1-5$ \\
\hline Nov & 0 & 0 & $1-5$ & $1-5$ & 0 & $\begin{array}{c}1 C-15 \mathrm{~N} \\
1-10 \mathrm{~S}\end{array}$ & $5-15$ & $1-5$ & $0-5$ & $1-5$ \\
\hline DEC & 0 & $i-2$ & $\begin{array}{c}D W \\
1-5 E\end{array}$ & $1-5$ & 0 & $\begin{array}{r}10-15 \mathrm{~N} \\
5-10 \mathrm{~S}\end{array}$ & $1-5$ & $1-5$ & 0 & $1-5$ \\
\hline
\end{tabular}

* 0 = few or rone. 
TABLE V-3

PERCENTAGE OF IVINDS WITH BEAUFORT FORCE 8 AND HIGHER

( $\geq 17.5$ meters per second)

\begin{tabular}{|c|c|c|c|c|}
\hline LOCATION & Dec-Jan-Feb & Mar-Apr-May & Jun-Jul-Aug . & Sep-Oct-Nov" \\
\hline Ivory Coast & 0 & 1 & 0 & 0 \\
\hline Mombasa & $\begin{array}{ll}N & 1 \\
S & 0\end{array}$ & 0 & 0 & 0 \\
\hline Sri Lanka & 0 & 0 & 1 & 0 \\
\hline Jakarta & 1 & 1 & 0 & 1 \\
\hline Dampier Land & 1 & 1 & 0 & 0 \\
\hline Philippines & 5 & 0 & 1 & $1-5$ \\
\hline Guam & 0 & 1 & 1 & $1-5$ \\
\hline Off Mexico' & 1 & 1 & 0 & 1 \\
\hline $\begin{array}{l}\text { Plant Ship } \\
\text { Pacific }\end{array}$ & - & 1 & 0 & \\
\hline $\begin{array}{l}\text { Plant Ship } \\
\text { Caribbean }\end{array}$ & 1 & 0 & 1 & 0 \\
\hline
\end{tabular}


TABLE $\mathrm{V}-4$

PREDOMINANT SURFACE WIND DIRECTICN

\begin{tabular}{|c|c|c|c|c|c|c|c|c|c|c|}
\hline MONTH & момBASA & \begin{tabular}{|c|c|} 
SRT \\
LANKRA
\end{tabular} & $\begin{array}{c}\text { DAMPIER } \\
\text { LAND }\end{array}$ & JAKARTA & MANILA & GUAM & $\begin{array}{l}\text { MEXF } \\
\text { MEXICO }\end{array}$ & $\begin{array}{c}\text { PLANT } \\
\text { SHIP } \\
\text { (PACIFIC) }\end{array}$ & $\begin{array}{l}\text { PLANIP } \\
\text { SHAIP } \\
\text { (CARIBN) }\end{array}$ & $\begin{array}{l}\text { IVORY } \\
\text { COAST }\end{array}$ \\
\hline JAN & $\downarrow$ & $<$ & די & $\searrow$ & $\downarrow$ & $\swarrow$ & $\searrow$ & $\swarrow$ & $\swarrow$ & $\uparrow \nearrow$ \\
\hline FEB & $\downarrow$ & 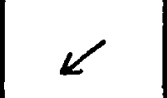 & $>$ & $\rightarrow$ & $<$ & $\swarrow$ & $\downarrow$ & $\measuredangle$ & $\measuredangle$ & $\uparrow$ \\
\hline MAR & $\leftarrow$ & $10 \%$ & $\longleftrightarrow$ & $\rightarrow$ & $\downarrow$ & $\downarrow$ & $\nu$ & $\measuredangle$ & $\leftarrow$ & $\uparrow$ \\
\hline${ }_{A P R}$ & $0^{\circ} \frac{\alpha}{\pi}$ & 0 & $k$ & $R$ & $\downarrow$ & $\leftarrow$ & $\searrow$ & $<$ & $\leftarrow$ & $\uparrow$ \\
\hline MAX & $\uparrow \uparrow$ & $\nearrow$ & $\pi$ & $\kappa$ & 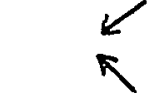 & $\angle$ & $\searrow$ & $\pi$ & $\leftarrow$ & $\uparrow$ \\
\hline JUN & $\therefore$ & $\lambda$ & $\pi$ & 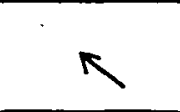 & $\lambda$ & $\leftarrow$ & $\nu$ & $x_{i} k$ & $\leftarrow$ & $\uparrow$ \\
\hline JUL & $\hat{\imath}$ & $\lambda$ & $\pi$ & $\pi$ & $\lambda$ & $\uparrow \leftarrow$ & \pm & $\uparrow$ & $\leftarrow$ & $\uparrow$ \\
\hline AUG & $\uparrow$ & $\pi$ & $\pi$ & $\pi$ & $\pi$ & $\leftarrow$ & $\downarrow$ & $\vec{\uparrow} \overrightarrow{ }$ & $\leftarrow$ & $>$ \\
\hline SEP & $\uparrow$ & $\rightarrow 0^{\lambda}$ & $\pi$ & $\pi$ & $v_{x}$ & $\leftarrow$ & $\nabla$ & $\nearrow$ & $\leftarrow$ & $\uparrow$ \\
\hline ост & $\uparrow$ & $\rightarrow$ & $\kappa$ & $\kappa$ & $<$ & $\swarrow$ & $\downarrow$ & $\uparrow$ & $\leftarrow$ & $\uparrow$ \\
\hline Nov & $\frac{\alpha}{\alpha}$ & $\rightarrow \infty$ & $\lambda$ & ${ }^{\Delta} \pi$ & $<$ & $\downarrow$ & $\downarrow$ & $k \downarrow$ & $\leftarrow$ & $\uparrow$ \\
\hline $\mathrm{DEC}^{\prime}$ & $\sigma$ & $\sigma$ & $\nearrow$ & $r$ & $\downarrow$ & $\downarrow$ & $\downarrow$ & $\leftarrow$ & $\leftarrow$ & $\lambda$ \\
\hline
\end{tabular}


VI. SEA AND SWELL CHARACTERISTICS

High sea and swell conditions present some problems to continuous OTEC operations for the area off Dampier Land. The area is within the main axis of the Southeast Trade Winds. High seas and swell are more common during the southern winter in July and August. Average frequencies (in percent) for the high and low seas and swells are presented in Table VI-1.

TABLE VI-I: SEA AND SWELL FREQUENCIES OFF DAMPIER LAND

\begin{tabular}{|l|c|c|}
\hline & $\begin{array}{c}\text { Low Seas } \\
(0.3-0.9 \text { meters })\end{array}$ & $\begin{array}{c}\text { High Seas } \\
\end{array}$ \\
\hline JAN-FEB & $42 \%$ & $8 \%$ \\
JUL-AUG & $29 \%$ & $16 \%$ \\
\hline
\end{tabular}

\begin{tabular}{|l|c|c|}
\hline & $\begin{array}{c}\text { Low Swell } \\
(0.3-1.8 \text { meters })\end{array}$ & $\begin{array}{c}\text { High Swell } \\
\text { (3.0 meters and higher })\end{array}$ \\
\hline JAN-FEB & $47 \%$ \\
JUL-AUG & $32 \%$ & $18 \%$ \\
& $31 \%$ \\
\hline
\end{tabular}

The source of this information is the U.S. Hydrographic Office's Wind Waves at Sea, (1947) [5] .

Table VI-2 provides wave statistics for the Dampier Land site taken from Ocean Wave Statistics, (1967), [19]. This statistical breakdown shows the number of observations in various height verses period catagories. The summary is 
TABLE VI-2: STATISTIZAL BREAKDOWN SHOWING NUMBER OF SHIPS OBSERVATIONS OFF DAMPIER LAND, RUSTRALIA REPORTING HEIGHT/PERIOD

COMBINATIONS (ALL SEASONS). From Ocean Wave Statistics (1967), [19].

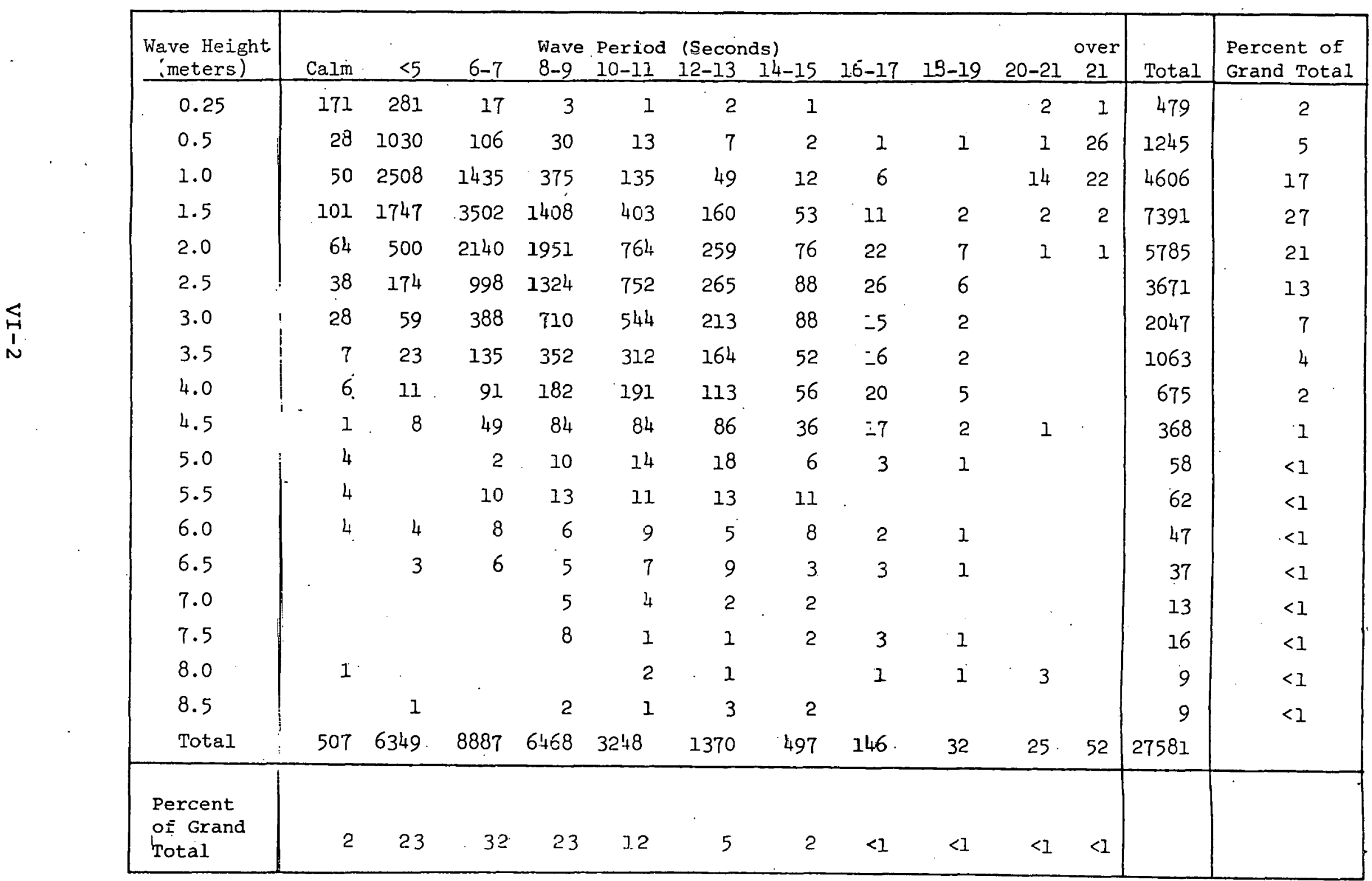


based upon actual ship's observations from a somewhat larger region than that for which the thermal resource was examined; however, they are generally representative of the. site region. Figure VI-1 provides a graph of the relative frequency of various wave heights for this site. 


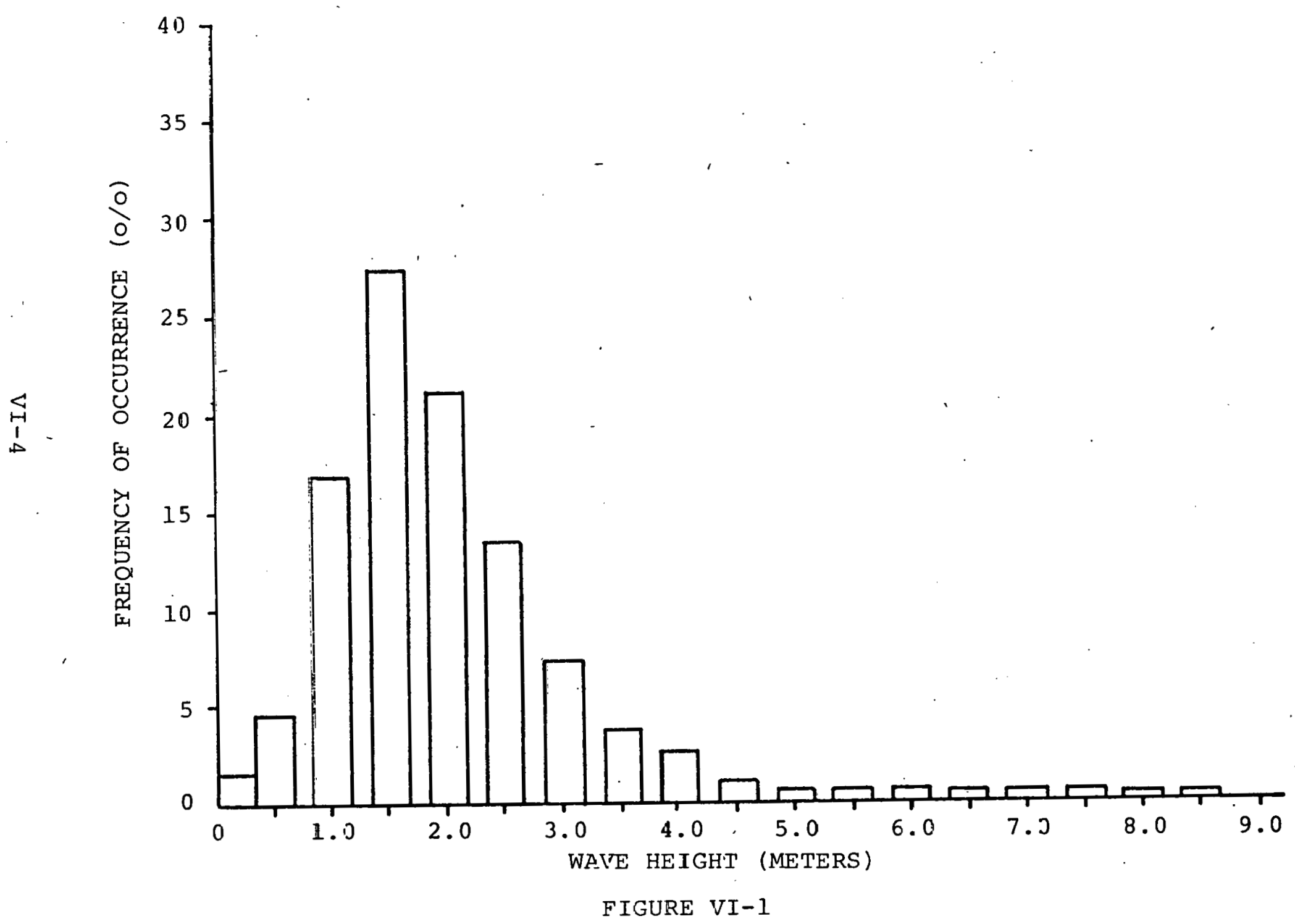

WAVE HEIGHT FREQUENCIES (DAMPIER LAND) 
VII. CURRENTS

Düing (1970, [14]) in the Monsoon Regime of the Currents in the Indian Ocean states the monsoon affected currents winds are generally limited to the area north of $10^{\circ} \mathrm{S}$. While the Dampier. Land site is south of $10^{\circ} \mathrm{S}$, the surface currents have the characteristic of a monsoon area in that the mean resultant surface current changes by more than $90^{\circ}$ through the year.

The circulation pattern of this region shows variation during the year. During the summer, the northward flowing cooler West Australia Current enters the area. The influence of the West Australia Current decreases in early winter. Subsequently, the South Equatorial Current penetrates the area with westward flowing waters, including some of Pacific origin. The monsoonal effects exist within the upper 200300 meters off Dampier Land (Düing, 1970, [14]). Surface current speeds are weak to moderate throughout the year with speeds generallý less than $51 \mathrm{~cm} / \mathrm{sec}$. Düing (1970, [14]). felt there was insufficient data for monthly charts of the currents in the Indian Ocean. Most of the knowledge of surface currents for this area is based on ship logs derived from calculations of ship's drift.

Hurricanes occasionally effect this region and can cause changes in the current regime. Leipper's (1967, [21]) study of Hurricane Hilda showed that a current had developed in the area transversed by the storm. Analysis of temperature-depth data showed a current of approximately $50 \mathrm{~cm} / \mathrm{sec}$. 
A theoretical study by O'Brien and Reid (1967, [27]) states that hurricanes will cause currents with a speed of approximately one meter per second.

The passage of hurricanes will induce upwellings of sub-surface waters for a temporary period of up to a week. This upwelling will cause anomalous vertical current shears. Additionally, Colburn and others have noted regions of upwelling off northwest Australia that may cause similar vertical current shears. The reqion between Java and the northwest coast of Australia is the source of the South. Equatorial Current of the Indian Ocean. Off the northwest coast of Australia the flow is generally offshore. An article by K. Wyrtki (1972, [45]) indicates that while there is fairly low temperature at 200 meters and the area is very rich in zooplankton, characteristics of upwelling, the strongest upwelling, is not along the Northwestern Australian shelf but along the coast of Java. Part of the reason for the rich zooplankton may be the strong tidal currents which effect the area. Still there is upwelling in this site region, which may cause strong current shears. 
1. Adamec, D., and O'Brien, J.J., November 1978, "The Seasonal Upwelling in the Gulf of Guinea Due to Remote Forcing", J: Phys. Oceanogr., 8, No. 6, 1050-1060.

2. Atwood, D.K., et al., 1976, Ocean Thermal Energy Conversion, Resource Assessment and Environment Impact for Proposed Puerto Rico Site, University of Puerto Rico.

3. Avery, W.H., et al., 1976, Maritime and Construction Aspects of Ocean Thermal Energy Conversion (OTEC) Plant Ships, The John Hopkins University Applied Physics Laboratory, Laurel, Maryland.

4. Bathen, K.H., et al., 1977, "Consolidated Oceanographic and Meteorological Data for Four North Pacific OTEC Sites", University of Hawaii.

5. Bigelow, H.B., and W.T. Emundson, 1947, Wind Waves at Sea, U.S. Hydrographic Office, Washington, D.C.

6. Colburn, J.G., 1974, "The Thermal structure of the Indian Ocean", International Indian Ocean Expedition Oceanographic Monograph No., 2; The University Press of Hawaii; Honolulu, Hawaii.

7. Crutcher, H.L. and R.G. Quayle, 1974, "Mariners Worldwide Climatic Guide to Tropical Storms at sea", NAVAIR 50lC-6I, Naval Weather Service; Asheville, N.C.

8. Defense Mapping Agency Hydrographic/Topographic Center, 1978, Pilot Chart of the North Pacific, DMA Stock Numbers PILOT 557801, 557804, 557810, Washington, D.C.

9. Defense Mapping Agency Hydrographic/Topographic Center, 1978, Pilot Chart of the North Atlantic Ocean, DMA Stock Numbers PILOT 167801; 167804, 167807, 167810, Washington, D.C.

10. Defense Mapping Agency Hydrographic Center, South Pacific Ocean, Sheet IV, Pub. IN.O. 623, revised 1974, Washington, D.C.

11. Defense Mapping Agency Hydrographic Center, Indian Ocean, Northern Part, Pub No. 721, 1974, Washington, D.C.

12. Defense Mapping Agency Hydrographic Center, Gulf of Mexico and Carribean Sea, Pub. No. 410, revised 1973, Washington, D.C. 
13. Defense Mapping Agency Hydrographic Center, North Atlantic Ocean, Southeastern Sheet, Publication N.O. 125, 4th ed., 1974, Washington, D.C.

14. Düing, W., 1970; "The Monsoon Regime of the Currents in the Indian Ocean", International Indian Ocean Expedition Oceanographic Monograph No. 1, Hawaii Institute of Geophysics Contribution No. 331, the University of Hawaii Press; Honolulu, Hawaii.

15. Düing, W. and F. Schott; March 1978, "Measurements in the Source Region of the Somali Current during the Monsoon Reversal", J. Phys. Oceanogr., 8, 278-289.

16. Emilsson, J., 1970, "On the Upper Layer Circulation in the Cayman Sea", from the symposium on the Investigations and Resources of the Caribbean Sea and Adjacent Regions, UNESCO, Palis Flallite.

17. Fleet Numerical Weather Central (FNWC), FNWC and National - Oceanographic Data Center digitized reports.

18. Gentry, R.C., 1970, "Hurricanes, One of the Major Features of Air-Sea Interaction in the Caribbean Sea", from the Symposium on the Investigation and Resources of the Caribbean Sea and Adjacent Regions, UNESCO, Paris, France.

19. Hogben, N., and F. Lumb, 1967, Ocean Wave Statistics, National Physical Laboratory, Ministry of Technology, London. Her Majesty's Stationery Office.

20. Johnson, A. and S. Denwick, 1978; "Data Report Buoy Observations During Hurricanes Anita and Babe, AugustSeptember, $14 \% \%$, NOAA Data Buoy Offioc, National Epacc Technology Laboratories, NSTL Station; Mississippi.

21. Leipper, D.F., 1967, "Observed Ocean Conditions and Furricane Hilda, 1964. J. Atmos Sci., 24, p 182-196.

22. McFadden J.D., "Airborne Investigations of the Effects of Hurricanes on the Thermal structure of the Surface Layer of the Ocean", from the Symposium on Investigation and Resources of the Caribbean Sea and Adjacent Regions, UNESCO, Paris France.

23. National Oceanographic Data Center, The Variability of Water Masses in the Indian Ocean, Publication G-11, Washington, D.C. 
24: National Oceanographic Data Center, Data File BTG75A, Washington, D.C., 1977.

25. National Oceanographic Data Center, Data Files SD40A5/0A6 from SD76A (1/30/76) 3509 STA: Washington, D.C., 1977.

26. National Science Foundation, 1972, Meteorological Atlas of the International Indian Ocean Expedition, Volume 7, Washington, D.C.

27. O'Brien, J.J., and R.O. Reid, 1967, "The Non-Linear Response of a Two Layer Baroclinic Ocean to a Stationery Axially Symetric Hurricane", J. Atmos. Sci., 24, p 197215 .

28. Ramage, C.D., 1972, "Indian Ocean Surface Meteorology", International Indian Ocean Expedition, Collective Reprints VIII, Contribution No. 624, pgs 407-540; Paris, France.

29. Sirvastava, P.S., P.K. Vyayarayou and M.X. Joseph, 1972

"Monthly Wave Characteristics of the Bay of Bengal", International Indian Ocean Expedition, Collective Reprints VIII, Contribution No. 625, Paris, France.

30. The Enclyopedia of Oceanography, 1966, Reinhold Publishing Corp., New York, New York.

31. U.S. Department of Agriculture, Weather Bureau, 19.38, "Atlas of Climatic Charts of the Oceans"., Washington, D.C.

32. U.S. Department of Commerce, NOAA, 1971, Eastropac Atlas, Volume 3, Washington, D.C.

33. U.S. Navy, 1975, Marine Climatic Atlas of the World, Volume III, Indian Ocean, Washington, D.C.

34. U.S. Navy Hydrographic Office, 1964, Atlas of Sea and Swell Charts, Northeastern Pacific Ocean, Publication No. 799D, revised 1976, Washington, D.C.

35. U.S. Naval Weather Service, September 1974, Climatic Summaries For Major Indian Ocean Ports and Waters; NAVAIR 50-1C-63, Asheville, N.C.

36. U.S. Naval Weather Service, November 1973, Climatic Summaries For Major Seventh Fleet Ports and Waters, NAVAIR 50-1C-62, Asheville, N.C.

37. U.S. Naval Oceanographic office, 1963, Oceanographic Atlas of the North Atlantic Ocean, Washington, D.C. 
38. U.S. Naval Oceanographic Office, Bathymetric Atlas of the Northeastern Pacific Ocean, Pub No. 1303-S, No. $0902 \mathrm{~N}, 0903 \mathrm{~N}, 1002 \mathrm{~N}, 1003 \mathrm{~N}$, Washington, D.C.

39. U.S. Naval Oceanographic Office, Indian Ocean, AfricaEast Coast Publication No. 724, lst ed., 1974, Washington, D.C.

40. U.S. Naval Oceanographic Office, 1971, Eathymetric Atlas of the Northeastern Pacific Ocean, H.O. Pub No. $1303-S, N .0 .1104 \mathrm{~N}, 1105 \mathrm{~N}, 1204 \mathrm{~N}, 1204 \mathrm{~N}$, Washington, D.C.

41. U.S. Naval Oceanographic Office, 1971, Bathymetric Atlas of the Northeastern Pacific Ocean, H.O. Pub N.O. $1301-S, 2403 \mathrm{~N}, 2040 \mathrm{~N}$, Washington, D.C.

42. U.S. Naval Oceanographic orfice, 1970, Bathymetric Atlas of the Northwestern Pacific Ocean, H.O. Pub. 1201-S, N.O. 2203N, Washington, D.C.

43. Wolff, P.M. and W.E. Hubert, 1976, "Ocean Thermal Energy Conversion: Resource, Ecological and Environmental Studies", Contract No. NSF-C1020, Ocean Data Systems, Inc., Monterey, California.

44. Wolff, P.M., et al, 1977., "OTEC Resource Report for Hawaii", Contract EG-77-C-01-4028, Ocean Data Systems, Inc., Monterey, California.

45. Wyrtki, K., 1972, "The Upwelling in the Region Between Java and Australia during the South-East Monsoon, Collected reprints of the International Indian Ocean Expedition, Volume I, Contribution No. 15, pp 151-161.

46. Wyrtki, K, 1972, "Geopotential Topographics and Assuciated Circulation in the South Eastern Indian Ucean", Collected reprints of the International Indan Ocean Expedition, Volume $I$, contribution No. 14 .

47. Wyrtki, K., 1971, Oceanographic Atlas of the International Ocean Expedition, National Science Foundation, NSF-IOE1, Washington, D.C. 